Recepción: 20 / 03 / 2019

Ciencias de la Ingeniería

Aceptación: 19 / 05 / 2019

Publicación: 05 / 06 / 2019

Artículo de Revisión

\title{
Implementación de un prototipo de estación de monitoreo (EM) para el análisis de variables medioambientales. Caso de estudio: Río Yanuncay de la ciudad de Cuenca
}

Implementation of a prototype monitoring station (EM) for the analysis of environmental variables. Case study: Río Yanuncay in the city of Cuenca

Implantação de um protótipo de estação de monitoramento (EM) para análise de variáveis ambientais. Estudo de caso: Río Yanuncay na cidade de Cuenca

\author{
Armando D. Chulde-Fuentes ${ }^{\text {I }}$ \\ danielos27@yahoo.com \\ Javier B. Cabrera-Mejía ${ }^{\text {II }}$ \\ jcabreram@ucacue.edu.ec
}

Correspondencia: danielos27@yahoo.com

I. Ingeniero en Electrónica y Telecomunicaciones, Dependencia de Posgrados. Universidad Católica de Cuenca, Cuenca, Ecuador.

II. Ingeniero Electrónico, Docente de la Unidad Académica de Ingeniería, Industria y Construcción de la Universidad Católica de Cuenca, Dependencia de Posgrados, Cuenca, Ecuador. 


\title{
Resumen
}

El presente trabajo pretende evaluar la operatividad de sistemas embebidos de open source, específicamente en la implementación de un prototipo de una estación de monitoreo de variables medioambientales, para determinar la calidad del agua, mismo que se pretende implementar en el Río Yanuncay de la ciudad de Cuenca. Para esto se utilizará sensores que permitan la adquisición de información de parámetros específicos, como: temperatura y turbidez del agua, niveles de potencial de óxido reducción (ORP) y de potencial de hidrógeno $(\mathrm{pH})$; dichos sensores se conectaran a una placa de desarrollo MEGA 2560 y esta a su vez a un módulo de comunicación inalámbrico, que garantice una conectividad continua y confiable de la información recolectada.

Para el diseño de la red de comunicación se realiza un análisis preliminar de los diferentes tipos de redes inalámbricas y los niveles de intensidad de campo (Cobertura) de las bandas de frecuencias asignadas a los diferentes sistemas de comunicación, puntualmente en los lugares donde se pretenderán emplazar los prototipos de estaciones de monitoreo, con el objetivo de determinar el medio de transmisión (medios de comunicación inalámbricos) más óptimo y eficiente, respetando la normativa existente y evitando producir interferencias perjudiciales a otros sistemas que ocupan el espectro radioeléctrico. Además, que se toma en consideración la topología de la ciudad de Cuenca, el cauce del Rio Yanuncay, la cobertura del servicio móvil avanzado (SMA) y de proveedores de servicios de acceso a internet.

Palabras claves: Placas de Desarrollo; Redes Inalámbricas; Sistemas de Monitoreo; Calidad del Agua; Sensores; Contaminación del Agua.

\begin{abstract}
The present work intends to evaluate the operation of embedded open source systems; it has been implemented as a prototype of a monitoring station for environmental variables to determine the water quality, which is implemented in the Yanuncay River of the city of Cuenca. To do this, use the sensors that allow the acquisition of them. The temperature and turbidity of the water, the levels of oxide reduction potential (ORP) and the hydrogen potential $(\mathrm{pH})$, will be presented: these sensors will be connected to an Arduino MEGA 2560 development board and this is once a wireless communication module, which guarantees a continuous and reliable connectivity of the information collected
\end{abstract}


The design of the communication network involves a preliminary analysis of the different types of wireless networks and the percentage of occupancy of the frequency bands assigned to the different communication systems, specifically in the places where the prototypes of radio stations are to be located. monitoring, with the objective of determining the most optimal and efficient means of transmission (wireless means of communication), respecting the existing legality and avoiding harmful interference to other systems that occupy the radioelectric spectrum. In addition, we refer to the topology of the city of Cuenca, the Yanuncay River, the advanced mobile service (SMA) coverage and the Internet access service providers.

Keys words: Development Plates; Wireless networks; Monitoring Systems; Water quality; Sensors; Water contamination.

\section{Resumo.}

Este trabalho tem como objetivo avaliar o funcionamento de sistemas embarcados open source, especialmente na implementação de um protótipo de uma estação de monitoramento variáveis ambientais para determinar a qualidade da água, que se destina a implementar no Yanuncay River City Cuenca. Para esta sensores capazes de adquirir informação parâmetros específicos será usada como temperatura e turbidez, níveis reduzidos de óxido de potencial (ORP) e potencial de hidrogénio $(\mathrm{pH})$; esses sensores serão conectados a uma placa de desenvolvimento MEGA 2560 e, por sua vez, a um módulo de comunicação sem fio, que garante uma conectividade contínua e confiável das informações coletadas.

Para o projeto da rede de comunicação uma análise preliminar dos diferentes tipos de redes sem fio e níveis de intensidade do campo (cobertura) das faixas de frequências atribuídas aos diferentes sistemas de comunicação, é realizado prontamente nos lugares onde eles fingem implantar estações de monitorização protótipo a fim de determinar o meio de transmissão (meios sem fios) ideal e eficiente, respeitando a legislação existente e evitando causa interferência prejudicial para outros sistemas de ocupação do espectro radioeléctrico. Além disso, leva em conta a topologia da cidade de Cuenca, o rio Yanuncay, a cobertura de móveis avançados (SMA) prestadores de serviços e acesso à Internet.

Palavras chaves: Placas de Desenvolvimento; Redes sem fio; Sistemas de Monitorização; Qualidade da Água; Sensores; Contaminação da água. 


\section{Introducción.}

La Constitución del Ecuador en sus artículos 12 y 318 señala que: “el agua constituye patrimonio nacional estratégico de uso público, inalienable, imprescriptible, inembargable y esencial para la vida”. Además, que “el Estado a través de la Autoridad Única del Agua, será responsable directo de la planificación y gestión de los recursos hídricos que se destinarán a consumo humano y riego que garantizarán la soberanía alimentaria, caudal ecológico y actividades productivas”. La entidad que se encarga de velar por el cumplimiento de estas leyes en el país es la Secretaria del Agua (SENAGUA).

A nivel mundial son latentes los problemas ocasionados por el calentamiento global, el efecto invernadero y otras variables contaminantes; en el país y en la ciudad de Cuenca en concreto se ve la necesidad de realizar una investigación que permita conocer la realidad medioambiental y la calidad del agua de sus ríos, para lo cual se debe realizar estudios que permitan analizar la factibilidad de la implementación de sistemas que permitan conocer datos en tiempo real de variables como temperatura, oxigenación, turbidez y pH que satisfagan y beneficien a la sociedad tanto en calidad de vida como tecnológicamente.

La Organización Mundial de la Salud (OMS), afirma que el estar expuestos e ingerir agua contaminada, la salud de decenas de millones de personas puede estar en peligro a causa de la contaminación de las aguas superficiales, provocando enfermedades como: la diarrea, la arsenicosis, fluorosis, la esquistosomiasis, helmintiasis, malaria, dengue, la legionelosis, la sepsis, etc. (Organización Mundial de la Salud, 2019)

En la investigación realizada por María Fernanda Arce Moncada y Márlin Adrián Leiva Calderón sobre la calidad de agua de los Ríos de la ciudad de Loja, se puede indicar que los afluentes que atraviesan el casco urbano, presentan altos grados de contaminación, concluyendo que, en algunos puntos, especialmente en los lugares de descarga de aguas hervidas, el agua es totalmente inservible para cualquier uso (Arce Moncada \& Leiva Calderón, 2009)

En el Ecuador la Ley Orgánica de Recursos Hídricos, Usos y Aprovechamiento del Agua indica que "los artículos 12, 313 y 318 de la Constitución de la República, consagran el principio de que el agua es patrimonio nacional estratégico, de uso público, dominio inalienable, imprescriptible e inembargable del Estado y constituye un elemento vital para la naturaleza y para la existencia de los 
seres humanos, reservando para el Estado el derecho de administrar, regular, controlar y gestionar los sectores estratégicos, de conformidad con los principios de sostenibilidad ambiental, precaución, prevención y eficiencia (Ley Orgánica de Recursos Hídricos, Usos y Aprovechamiento del Agua, 2014)

Según la Norma de Calidad Ambiental y de Descargas de efluentes: Recurso Agua, en el anexo 1 del libro VI, se establece en el literal a "los límites permisibles, disposiciones y prohibiciones para las descargas en cuerpos de aguas o sistemas de alcantarillado”, para los diferentes usos del agua como: consumo humano y uso doméstico, Preservación de Flora y Fauna, Agrícola, Pecuario, Recreativo, Industrial, Transporte y Estético. (REFORMA DEL LIBRO VI DEL TEXTO UNIFICADO DE LEGISLACIÓN SECUNDARIA)

Para el presente estudio se toma los criterios admisibles para la preservación de la flora y fauna en aguas dulces, frías o cálidas, y en aguas marinas y de estudio, dados en la Norma de Calidad Ambiental y de Descarga de Efluentes.

Tabla 1 Criterios de Calidad admisibles para la preservación de la flora y fauna en aguas dulces

\begin{tabular}{|l|l|l|l|}
\hline Parámetro & Expresados como & Unidad & $\begin{array}{l}\text { Límite máximo } \\
\text { permisible }\end{array}$ \\
\hline Temperatura & ${ }^{\circ} \mathrm{C}$ & & $\begin{array}{l}\text { Condiciones naturales } \\
+3\end{array}$ \\
\hline Potencial de hidrógeno & $\mathrm{pH}$ & $\mathrm{UNT}$ & $6,5-9$ \\
\hline Turbiedad & & $\mathrm{mV}$ & 100 \\
\hline $\begin{array}{l}\text { Potencial de óxido } \\
\text { reducción }\end{array}$ & ORP & ------ \\
\hline
\end{tabular}

Fuente: (REFORMA DEL LIBRO VI DEL TEXTO UNIFICADO DE LEGISLACIÓN SECUNDARIA)

\section{Desarrollo.}

De acuerdo con lo que indica la guía para la calidad del agua de consumo humano en su cuarta edición, "La vigilancia es un elemento importante en el desarrollo de estrategias para la mejora gradual de la calidad de los servicios de abastecimiento de agua de consumo humano. Es importante desarrollar 
estrategias para implementar el programa de vigilancia, recopilar, analizar y resumir los datos, y notificar y diseminar los hallazgos, y que las estrategias vengan acompañadas de recomendaciones sobre medidas correctivas." (Organización Mundial de la Salud, 2018) . En este contexto se ve la necesidad de desarrollar sistemas de monitoreo en tiempo real de variables que garantizan la calidad del agua de uno de los principales Ríos de la ciudad de Cuenca, con el objetivo a que se lo pueda replicar a los demás afluentes, teniendo datos que den información de los niveles de las variables medioambientales y sobre todo ver los factores contaminantes que puedan estar afectando y realizar acciones correctivas y sancionatorias por parte de los entes reguladores.

El agua por ser el líquido vital para la supervivencia de los seres vivos, ha generado gran interés en la comunidad científica, por lo que se han concebido un sin número de proyectos e investigaciones para su preservación, gran cantidad de estos presentan características comunes, de acuerdo a (El-Khouri Vidarte, 2016), existen 3 grupos de proyectos:

- Proyectos Open Source/ Open Hardware: los códigos que se generan son libres, y se encuentra información completa de los dispositivos, sensores, hardware utilizado y diagrama de bloques del sistema, con el fin de que sea replicado y mejorado.

- Proyectos surgidos del ámbito académico: Por la necesidad de fomentar la investigación y el uso de nuevas tecnologías de bajo costo, se generan proyectos en algunos casos en colaboración con entes del estado y universidades, teniendo como base proyectos Open Source y Open Hardware.

- Proyectos colaborativos y multidisciplinarios: Por su complejidad y costo, este tipo de proyectos se suele dividir en bloques, manteniendo una buena comunicación para lograr los objetivos planteados.

Para el presente trabajo de investigación se tomó como base un proyecto del tipo académico, tomando en consideración la información existente, la disponibilidad de los diferentes sensores y placa de desarrollo de hardware, lo que implica su bajo costo y especialmente por lo que se alinea a los objetivos planteados

En el mercado existen varios sistemas de monitoreo de la calidad del agua tanto equipos portátiles, que su desventaja principal es que necesitan de la intervención de un técnico para la toma de muestras, 
lo que impiden tener datos continuos y el costo de operación se eleva ya que depende la frecuencia con la que se toman las muestras y equipos autónomos, los cuales tiene costos elevados.

En la página web oficial de la Agencia de Protección Ambiental de Estados Unidos, se muestran los datos en tiempo Real de variables físicas y químicas que dan la información necesaria que garantizar la calidad del agua de la cuenca baja del Río Merrimack (ubicado al noroeste de los Estados Unidos y fluye hacia el sur al estado de Massachusetts), e históricos de datos que se los puede descargar para su análisis. En este proyecto se utiliza una estación de monitoreo que toma datos cada 15 minutos de la temperatura, oxígeno disuelto, $\mathrm{pH}$, conductancia específica, turbidez, clorofila y ficocianina, estos sensores se encuentran a un metro por debajo de la superficie del agua, misma que es recolectada mediante una bomba y tubería aislada que toma el agua desde y la transporta a la estación de monitoreo tal como lo podemos ver en la figura 2, y la información receptada se transmite en tiempo real por medio de telemetría. (Agencia de Protección Ambiental de Estados Unidos, 2018)

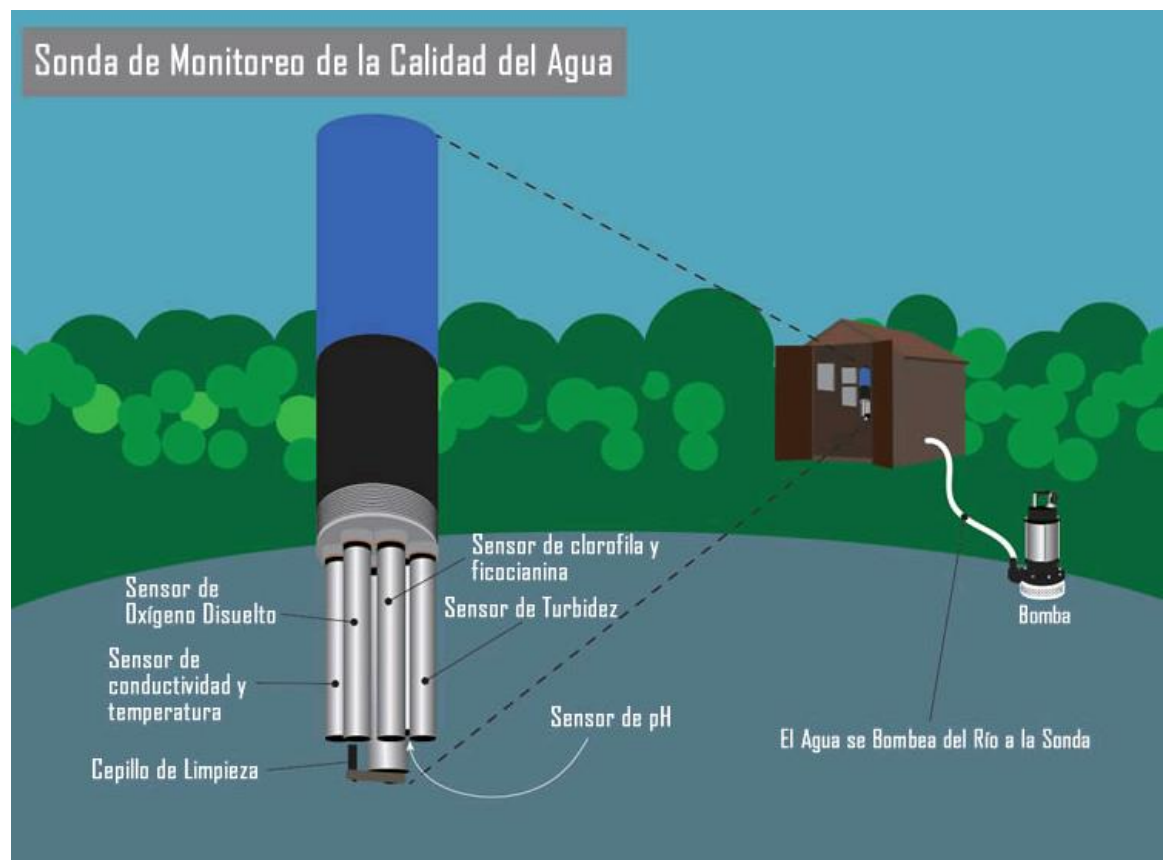

Figura 1.Proceso de adquisición y almacenamiento de la información obtenida Fuente: Agencia de Protección Ambiental de Estados Unidos 
Para este proyecto de investigación se toma como referencia la manera de recolección del agua del proyecto del Río Merrimack, ya que el caudal del Rio Yanuncay de la ciudad de Cuenca es impredecible, además por la seguridad e integridad de la estación de monitoreo a implementar.

Tomando en cuenta el uso de plataformas y placas de desarrollo con software libre para el proyecto y previo a un análisis en el que se consideró el alcance del proyecto, se utiliza los siguientes recursos para la implementación del prototipo de la estación de monitoreo:

El uso de software libre, placas de desarrollo de hardware, sensores, y un medio de comunicación confiable permitirá que el presente trabajo de investigación sea replicable a otras ciudades, aportando así a la sociedad con información que permitirá tomar conciencia de la importancia que conlleva el cuidar el agua siendo este el líquido vital La Organización Mundial de la Salud en lo referente a la salubridad y calidad del agua indica "La salubridad y la calidad del agua son fundamentales para el desarrollo y el bienestar humanos. Proporcionar acceso a agua salubre es uno de los instrumentos más eficaces para promover la salud y reducir la pobreza.” (Organización Mundial de la Salud, 2017) 
Implementación de un prototipo de estación de monitoreo (EM) para el análisis de variables medioambientales. Caso de estudio: Río Yanuncay de la ciudad de Cuenca

Tabla 2 Dispositivos utilizados para la elaboración del prototipo

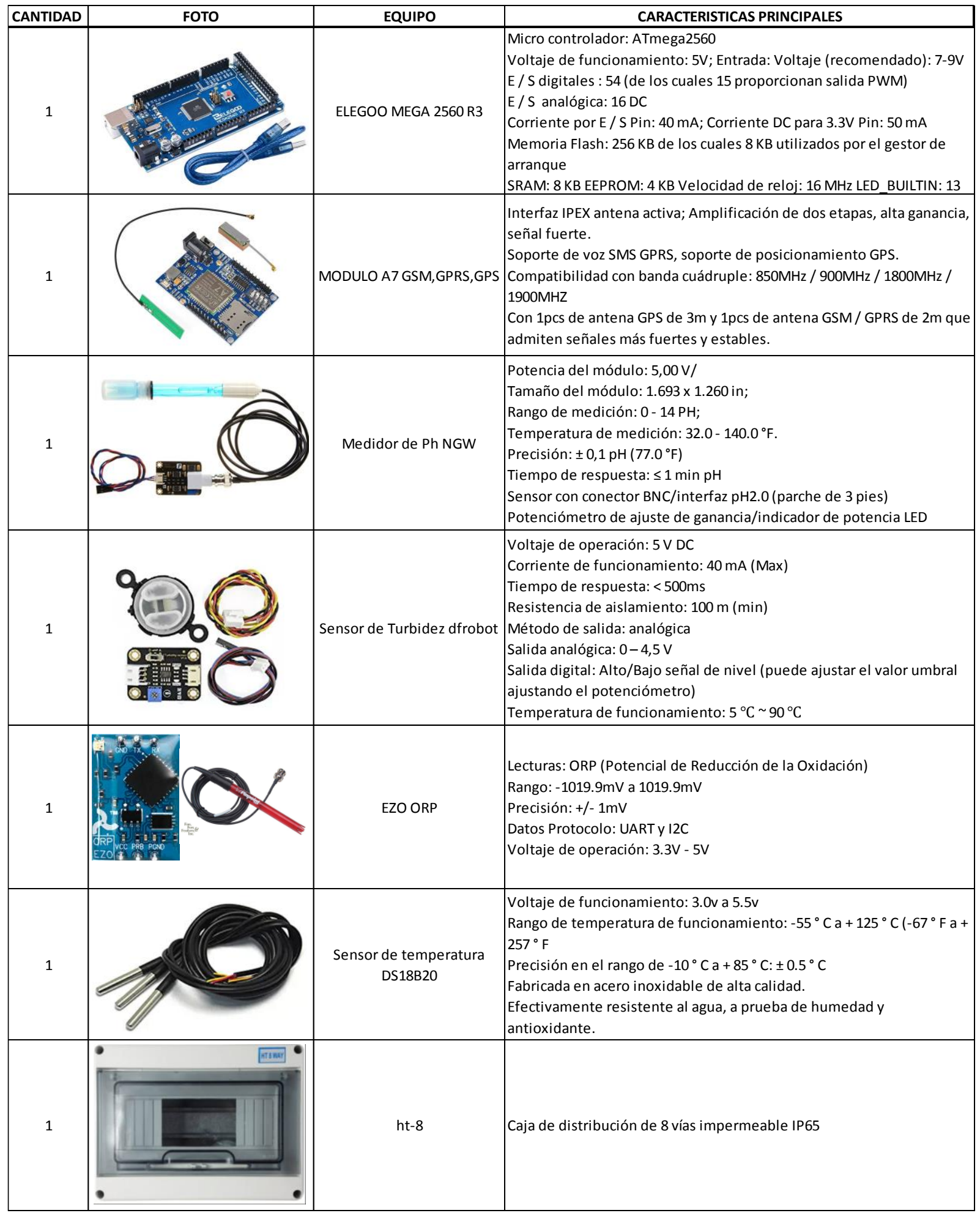

Fuente: (Amazon.com, 2019) 
Para el almacenamiento, procesamiento, análisis y presentación de la información se consideró utilizar la plataforma thinger.io, que es una plataforma de código abierto de fácil manejo y con un gran potencial en los referente al internet de las cosas, además que presta servicio de almacenamiento en la nube para acceder a la información de la lectura de los diferentes sensores en tiempo real y tener un histórico para realizar análisis de los valores obtenidos, esto permitirá conocer de manera precisa comportamientos inusuales en la calidad del agua del Rio Yanuncay, y servirán para tomar acciones correctivas por parte de los entes de regulación.

Una vez que se adquieren los datos por los sensores y son procesados en el microcontrolador Mega 2560 se envían los datos al internet, a través de la red de las operadora de servicio móvil avanzado que se ha determinado en el análisis de la red inalámbrica a utilizar, una vez que se autentica en los servidores de la plataforma IO, se crean las diferentes bases de datos una para cada variable y se tiene la posibilidad de generar interfaces de programación de aplicaciones (API), que pueden ser utilizadas en aplicaciones WEB .
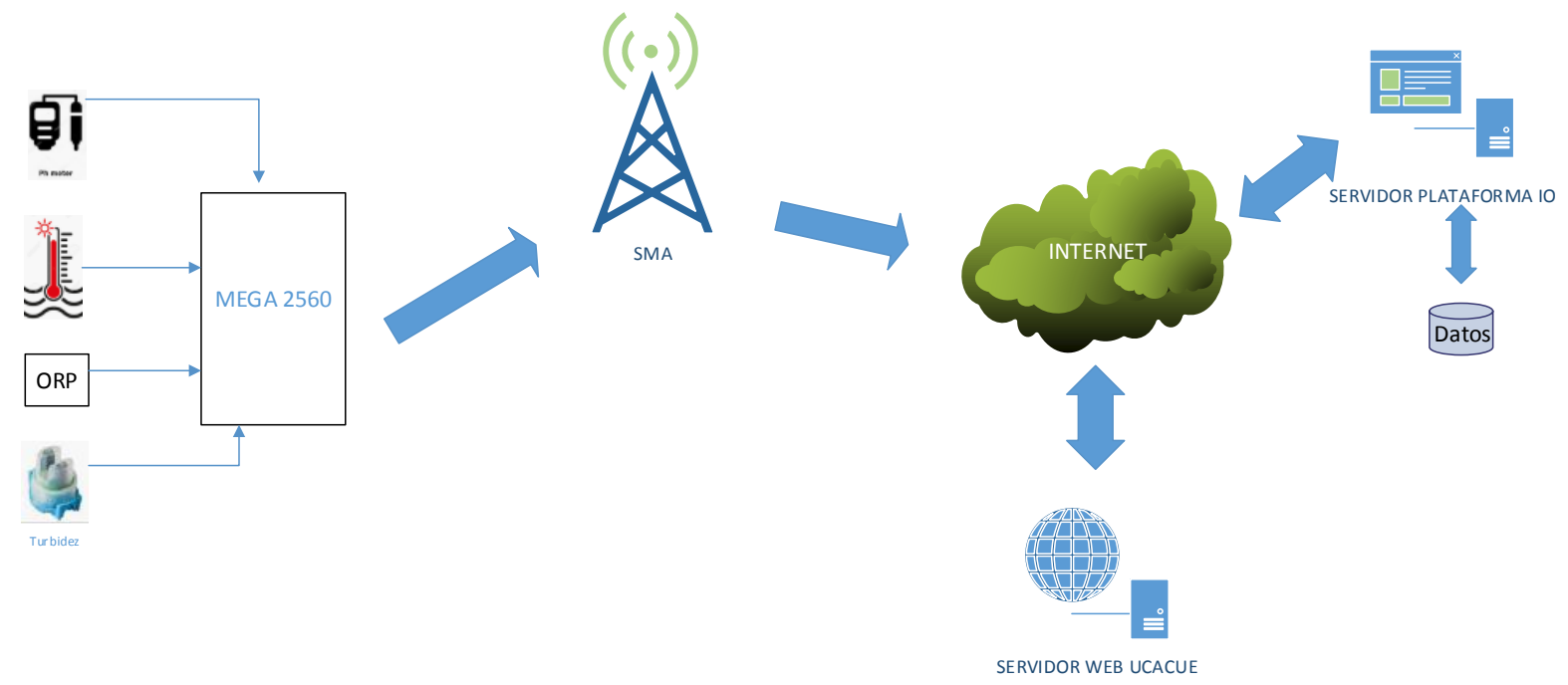

Figura 2. Diseño de la arquitectura de la Estación de Monitoreo (EM)

Fuente: Autoría Propia

Para el análisis de la tecnología inalámbrica y el proveedor de servicio de telecomunicaciones a utilizar, se realiza un monitoreo del espectro radioeléctrico con ayuda de un analizador marca NARDA modelo SRM-3006, proporcionado por la Agencia de Regulación y Control de las Telecomunicaciones (ARCOTEL), el cual tiene un rango de operación de $27 \mathrm{MHz}$ a $3 \mathrm{GHz}$. 
Implementación de un prototipo de estación de monitoreo (EM) para el análisis de variables medioambientales. Caso de estudio: Río Yanuncay de la ciudad de Cuenca

Los puntos tentativos para el emplazamiento de las estaciones de monitoreo del agua del Rio Yanuncay de la ciudad de Cuenca, se escogieron tomando en consideración aspectos como la seguridad y accesibilidad. Sus coordenadas geográficas se las detallan en la tabla 3, se consideró inicialmente tres puntos uno al ingreso de la ciudad, al centro y antes de que se unan los ríos y se convierta en el Rio Cuenca.

Tabla 3.Dispositivos utilizados para la elaboración del prototipo

\begin{tabular}{|c|c|c|c|c|}
\hline \multirow{2}{*}{ ID } & \multirow{2}{*}{ LUGAR } & \multirow{2}{*}{ UBICACIÓN } & \multicolumn{2}{|c|}{ COORDENADAS GEOGRÁFICAS } \\
\hline & & & LATITUD & LONGITUD \\
\hline EM1 & TRUJILLO PLAZA MARKET & $\begin{array}{l}\text { Medio Ejido, Cuenca - Molleturo - Naranjal, } \\
\text { Cuenca } 010116\end{array}$ & $2^{\circ} 54^{\prime} 5.50^{\prime \prime S}$ & $79^{\circ} 2 ' 48.90^{\prime \prime O}$ \\
\hline EM2 & $\begin{array}{c}\text { Benemerito Cuerpo de Bomberos } \\
\text { Voluntarios de Cuenca }\end{array}$ & Av. 27 de Febrero & $2^{\circ} 54^{\prime} 57.20^{\prime \prime} \mathrm{S}$ & $79^{\circ} 00^{\prime} 14.50^{\prime \prime} \mathrm{O}$ \\
\hline EM3 & Parque El Paraiso & Laguna del Parque el Paraiso & $2^{\circ} 54^{\prime} 43.60^{\prime \prime S}$ & $78^{\circ} 59^{\prime} 21.40^{\prime \prime} \mathrm{O}$ \\
\hline
\end{tabular}

Fuente: Autoría Propia

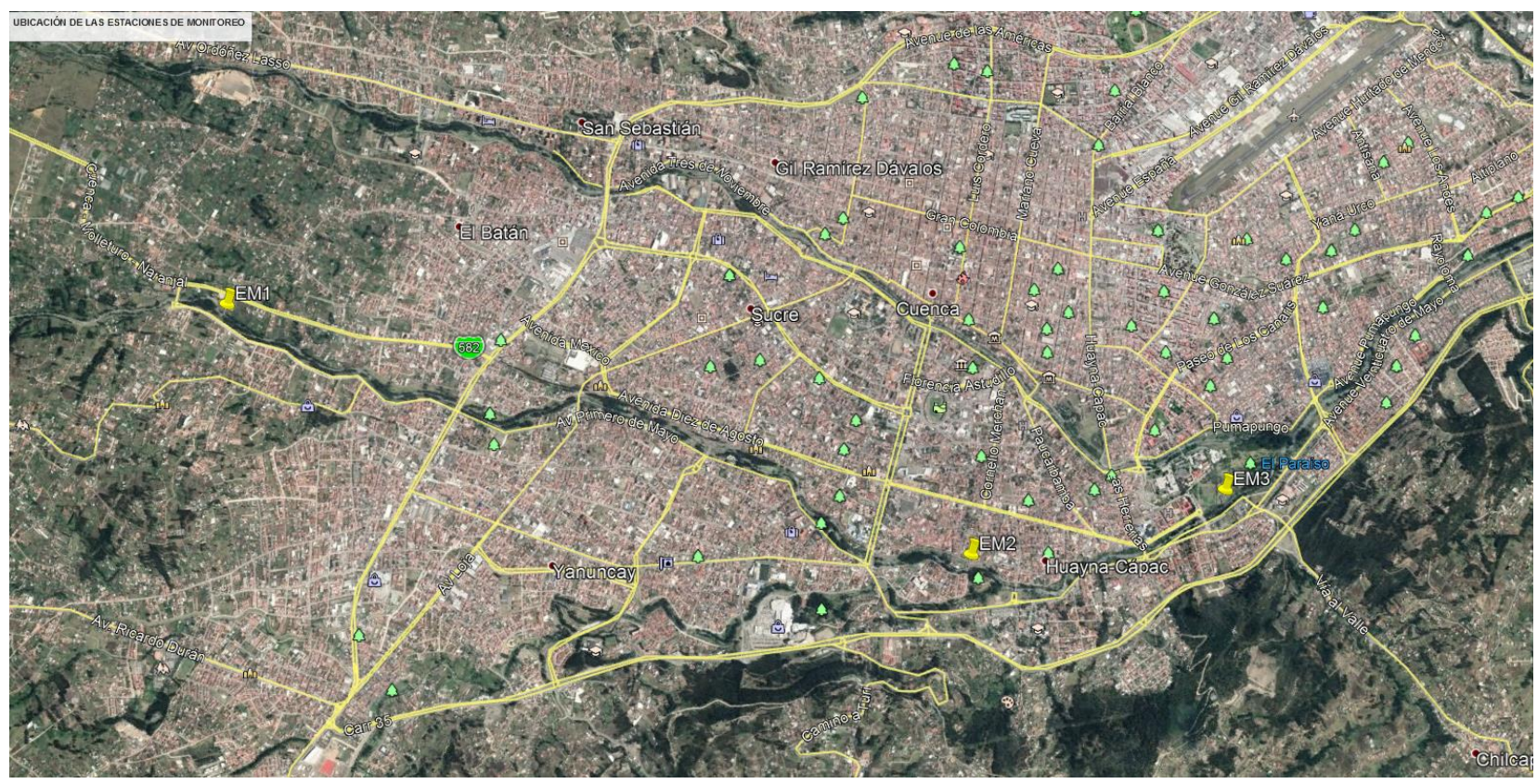

Figura 3. Ubicación geográfica de los posibles lugares de emplazamiento de las EM.

Fuente: Autoría Propia 


\section{Metodología.}

El desarrollo del presente trabajo de investigación se la realizará por fases, siendo como primera la recopilación de información de proyectos referentes a la implementación de estaciones de monitoreo de variables medioambientales, placas de desarrollo de hardware, sus ventajas, limitaciones, y un estudio del cauce del Rio Yanuncay para poder establecer la ubicación adecuada para el emplazamiento de la estación de monitoreo, considerando aspectos importantes como seguridad, fácil acceso, prevención de caudal, etc.

La segunda fase será el estudio de las redes de comunicación (Wifi, GSM,CDMA, GPRS, MDBA) que se encuentran disponibles en los puntos donde se emplazarán las estaciones de monitoreo, para que se garantice la fiabilidad y continuidad de los datos recolectados, para su procesamiento, almacenamiento y publicación en los portales web de la Universidad Católica de Cuenca, con el objetivo de que la información esté disponible para todos los interesados en el tema ya sea la población o entes de control y regulación.

Y la tercera fase será el desarrollo e implementación de un prototipo de estación de monitoreo de variables medioambientales que proporcione información de la calidad del agua del Rio Yanuncay de la ciudad de Cuenca, para lo cual se medirá temperatura, potencial de óxido reducción (ORP), turbidez y potencial de hidrogeno $(\mathrm{pH})$, con ayuda de sensores y sondas compatibles con placas de desarrollo de hardware y software (Arduino) y su evaluación en campo.

Como fase final se tendrá la verificación de la hipótesis planteada con las conclusiones y recomendaciones de los resultados obtenidos

\section{Resultados.}

\section{Análisis del espectro radioeléctrico.}

Se puede indicar que la tecnología inalámbrica de comunicación que nos garantizaría un continuo flujo de la información entre la estación de monitoreo y los diferentes servidores de almacenamiento y procesamiento, es el servicio móvil avanzado, además de que es un servicio regulado y controlado por la ARCOTEL, garantizaría que los datos obtenidos por los diferentes sensores y procesados en el microcontrolador Mega 2500, se transmitan de una manera segura y legal al servidor de la plataforma 
Implementación de un prototipo de estación de monitoreo (EM) para el análisis de variables medioambientales. Caso de estudio: Río Yanuncay de la ciudad de Cuenca

IO, y se evitaría la concesión, o uso ilegal del espectro radioeléctrico y causar interferencias perjudiciales a otros sistemas de telecomunicaciones.

Para el punto (EM1), ubicado en el sector de Misicata (parte posterior del almacén Trujillo Plaza Market), se obtiene los siguientes resultados.

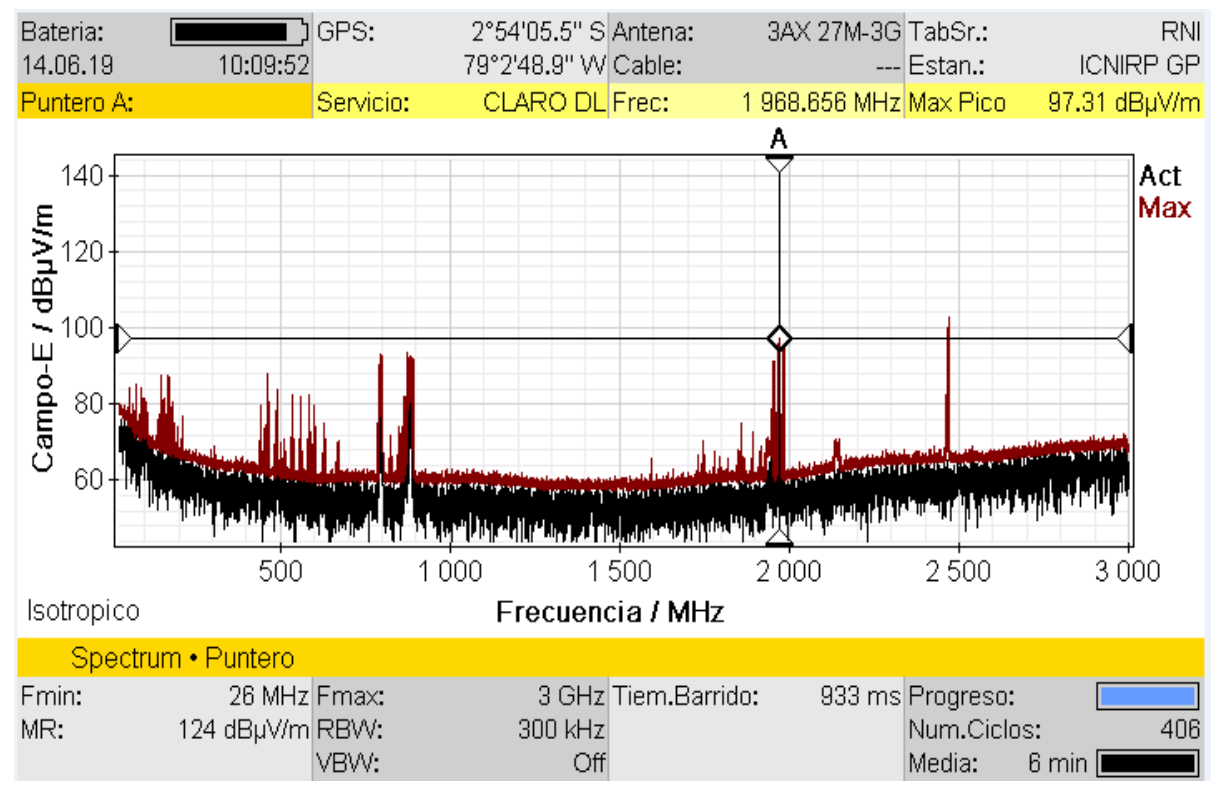

Figura 4. Captura Espectral en EMI

Fuente: Autoría Propia

\begin{tabular}{|c|c|c|c|c|c|}
\hline $\begin{array}{l}\text { Bateria: } \\
14.06 .19\end{array}$ & $\square$ GPS: & \multicolumn{2}{|c|}{$\begin{array}{l}2^{\circ} 54^{\prime} 05.6^{\prime \prime} \text { S Antena: } \\
\text { 79 2'48.8" W Cable: }\end{array}$} & $\begin{array}{r}\text { 3AX 27M-3G TabSr.: } \\
\text {--- Estan.: }\end{array}$ & $\begin{array}{r}\text { RNI } \\
\text { ICNIRP GP }\end{array}$ \\
\hline \multicolumn{5}{|c|}{ Tabla de Picos } & \\
\hline ndice & Frecuencia & Servicio & Maximo & Activar & \\
\hline 1 & $2469.502826 \mathrm{MHz}$ & & $103.21 \mathrm{~dB} \mu \mathrm{V} / \mathrm{m}$ & $-43.57 \mathrm{~dB}$ & \\
\hline 2 & $2468.500439 \mathrm{MHz}$ & & $100.54 \mathrm{~dB} \mu \mathrm{V} / \mathrm{m}$ & $-43.04 \mathrm{~dB}$ & \\
\hline 3 & $2467.476754 \mathrm{MHz}$ & & $98.37 \mathrm{~dB} \mu \mathrm{V} / \mathrm{m}$ & $-40.35 \mathrm{~dB}$ & \\
\hline 4 & $1968.655922 \mathrm{MHz}$ & CLARO DL & $97.31 \mathrm{~dB} \mu \mathrm{V} / \mathrm{m}$ & $-18.57 \mathrm{~dB}$ & \\
\hline 5 & $1966.941676 \mathrm{MHz}$ & CLARO DL & $95.43 \mathrm{dB \mu} / \mathrm{m}$ & $-5.62 \mathrm{~dB}$ & \\
\hline 6 & $1981.653883 \mathrm{MHz}$ & CNT DL & $94.35 \mathrm{~dB} \mu \mathrm{V} / \mathrm{m}$ & $-17.96 \mathrm{~dB}$ & \\
\hline 7 & $1983.516414 \mathrm{MHz}$ & CNT DL & $94.33 \mathrm{~dB} \mu \mathrm{V} / \mathrm{m}$ & $-9.37 \mathrm{~dB}$ & \\
\hline 8 & $1967.629968 \mathrm{MHz}$ & CLARO DL & $94.08 \mathrm{~dB} \mu \mathrm{V} / \mathrm{m}$ & $-12.43 \mathrm{~dB}$ & \\
\hline 9 & $875.911459 \mathrm{MHz}$ & CLARO DL & $93.79 \mathrm{~dB} \mu \mathrm{V} / \mathrm{m}$ & $-15.17 \mathrm{~dB}$ & \\
\hline 10 & $1982.354220 \mathrm{MHz}$ & CNT DL & $93.72 \mathrm{~dB} \mu \mathrm{V} / \mathrm{m}$ & $-20.84 \mathrm{~dB}$ & \\
\hline 11 & $1965.954220 \mathrm{MHz}$ & CLARO DL & $93.44 \mathrm{~dB} \mu \mathrm{V} / \mathrm{m}$ & $-16.38 \mathrm{~dB}$ & \\
\hline \multicolumn{6}{|c|}{ Isotropico } \\
\hline \multicolumn{6}{|c|}{ Spectrum • Tabla de Picos } \\
\hline \multirow{3}{*}{$\begin{array}{l}\text { Fmin: } \\
\text { MR: }\end{array}$} & \multirow{3}{*}{\multicolumn{3}{|c|}{$\begin{array}{r}3 \mathrm{GHz} \mathrm{T} \\
300 \mathrm{kHz} \\
\text { Off }\end{array}$}} & \multirow{3}{*}{$\begin{array}{l}946 \text { ms Progreso: } \\
\text { Num.Ciclos: } \\
\text { Media: }\end{array}$} & \\
\hline & & & & & 426 \\
\hline & & & & & $6 \min \square$ \\
\hline
\end{tabular}

Figura 5. Evaluación del Espectro en el rango de $27 \mathrm{MHz}$ a $3 \mathrm{GHz}$ en EMI

Fuente: Autoría Propia 
El nivel de intensidad de campo mayor en el primer punto evaluado, corresponde a una red privada de Wifi, que opera en la banda de $2.4 \mathrm{GHz}$ : para nuestro proyecto con estos resultados podemos concluir que en ese sitio debemos utilizar el servicio de móvil avanzado de la operadora Claro, ya que las señales más fuertes corresponden a una radio base de esa operadora, lo que garantizaría la transmisión de la información hacia el servidor de la plataforma IO.

De la misma manera en el punto dos y tres se realiza el análisis de cobertura de las tres operadoras que brindan el servicio móvil avanzado

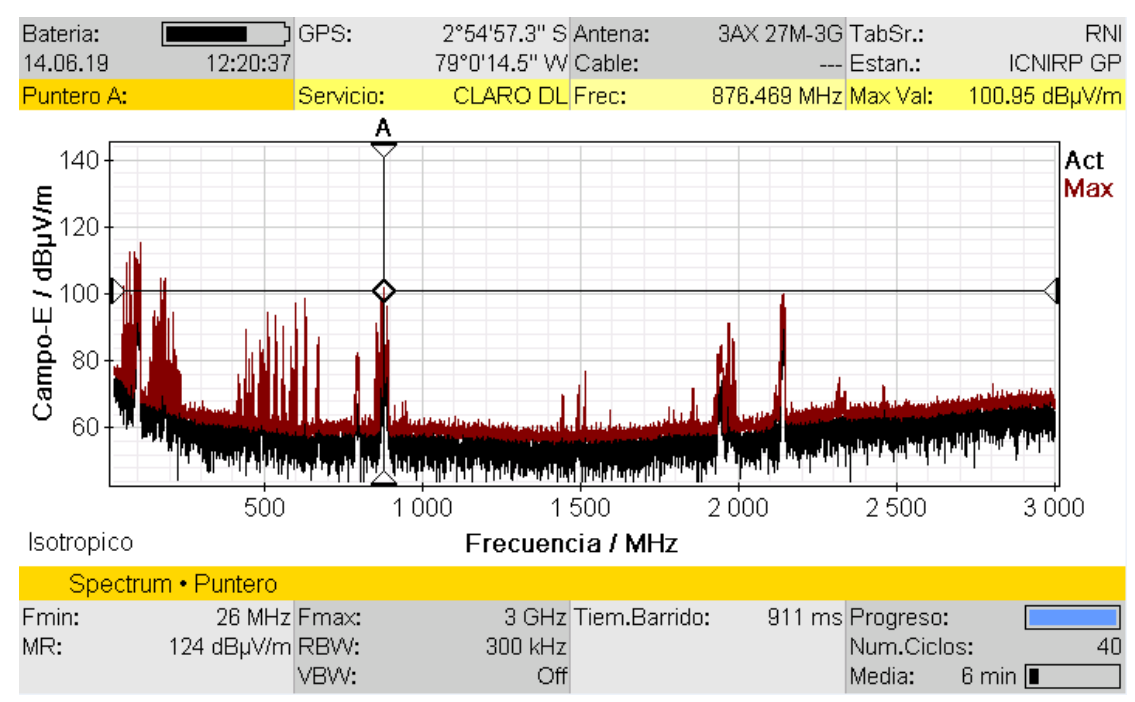

Figura 6. Captura Espectral en EM2

Fuente: Autoría Propia

\begin{tabular}{|c|c|c|c|c|c|}
\hline $\begin{array}{l}\text { Bateria: } \\
14.06 .19\end{array}$ & $\underset{12: 21: 10}{\longrightarrow}$ GPS & $\begin{array}{r}2^{\circ} 54^{\prime} 57.2^{\prime} \\
79^{\circ} 0^{\prime} 14.5^{\prime \prime}\end{array}$ & $\begin{array}{l}\text { Antena: } \\
\text { Cable: }\end{array}$ & $\begin{array}{r}\text { 7M-3G TabSr.: } \\
\text {--- Estan.: }\end{array}$ & $\begin{array}{r}\text { RNI } \\
\text { ICNIRP GP }\end{array}$ \\
\hline Tabla d & e Picos & & & & $\Delta \nabla$ \\
\hline ndice & Frecuencia & Servicio & Maximo & Activar & \\
\hline 23 & $101.670801 \mathrm{MHz}$ & & $103.92 \mathrm{~dB} \mu \mathrm{V} / \mathrm{m}$ & $-3.55 \mathrm{~dB}$ & \\
\hline 24 & $94.882978 \mathrm{MHz}$ & & $103.91 \mathrm{~dB} \mu \mathrm{V} / \mathrm{m}$ & $-1.23 \mathrm{~dB}$ & \\
\hline 25 & $104.899727 \mathrm{MHz}$ & & $102.98 \mathrm{~dB} \mu \mathrm{V} / \mathrm{m}$ & $-0.87 \mathrm{~dB}$ & \\
\hline 26 & $55.248490 \mathrm{MHz}$ & & $102.77 \mathrm{~dB} \mu \mathrm{V} / \mathrm{m}$ & $-3.06 \mathrm{~dB}$ & \\
\hline 27 & $876.567358 \mathrm{MHz}$ & CLARO DL & $102.14 \mathrm{~dB} \mu \mathrm{V} / \mathrm{m}$ & $-13.50 \mathrm{~dB}$ & \\
\hline 28 & $2132.298259 \mathrm{MHz}$ & CLARO AWS DL & $100.82 \mathrm{~dB} \mu \mathrm{V} / \mathrm{m}$ & $-13.47 \mathrm{~dB}$ & \\
\hline 29 & $2139.897598 \mathrm{MHz}$ & CLARO AWS DL & $100.21 \mathrm{~dB} \mu \mathrm{V} / \mathrm{m}$ & $-16.22 \mathrm{~dB}$ & \\
\hline 30 & $2140.165468 \mathrm{MHz}$ & CLARO AWS DL & $100.13 \mathrm{~dB} \mu \mathrm{V} / \mathrm{m}$ & $-13.32 \mathrm{~dB}$ & \\
\hline 31 & $2131.978315 \mathrm{MHz}$ & CLARO AWS DL & $99.35 \mathrm{~dB} \mu \mathrm{V} / \mathrm{m}$ & $-17.75 \mathrm{~dB}$ & \\
\hline 32 & $2131.485160 \mathrm{MHz}$ & CLARO AWS DL & $99.34 \mathrm{~dB} \mu \mathrm{V} / \mathrm{m}$ & $-12.39 \mathrm{~dB}$ & \\
\hline 33 & $627.251468 \mathrm{MHz}$ & & $98.70 \mathrm{~dB} \mu \mathrm{V} / \mathrm{m}$ & $-7.90 \mathrm{~dB}$ & \\
\hline
\end{tabular}

\begin{tabular}{|c|c|c|c|c|}
\hline \multicolumn{5}{|c|}{ Spectrum • Tabla de Picos } \\
\hline Fmin: & $26 \mathrm{MHz}$ Fmax: & $3 \mathrm{GHz}$ Tiem.Barrido: & 942 ms Progreso: & $\square$ \\
\hline MR: & 124 dB $\mu$ V/m RBW: & $300 \mathrm{kHz}$ & Num.Ciclos: & 74 \\
\hline & VBW: & Off & Media: $\quad 6 \min [$ & $\square$ \\
\hline
\end{tabular}

Figura 7. Evaluación del Espectro en el rango de $27 \mathrm{MHz}$ a $3 \mathrm{GHz}$ en EM2

Fuente: Autoría Propia 
Implementación de un prototipo de estación de monitoreo (EM) para el análisis de variables medioambientales. Caso de estudio: Río Yanuncay de la ciudad de Cuenca

En este sitio se puede apreciar que se tiene mayor intensidad de campo los servicios de radio difusión y televisión esto por estar en línea de vista con los transmisores de este servicio, pero la operadora con mayor nivel de intensidad de campo en el lugar es la operadora Claro.

En el último punto evaluado se obtuvieron los siguientes resultados:

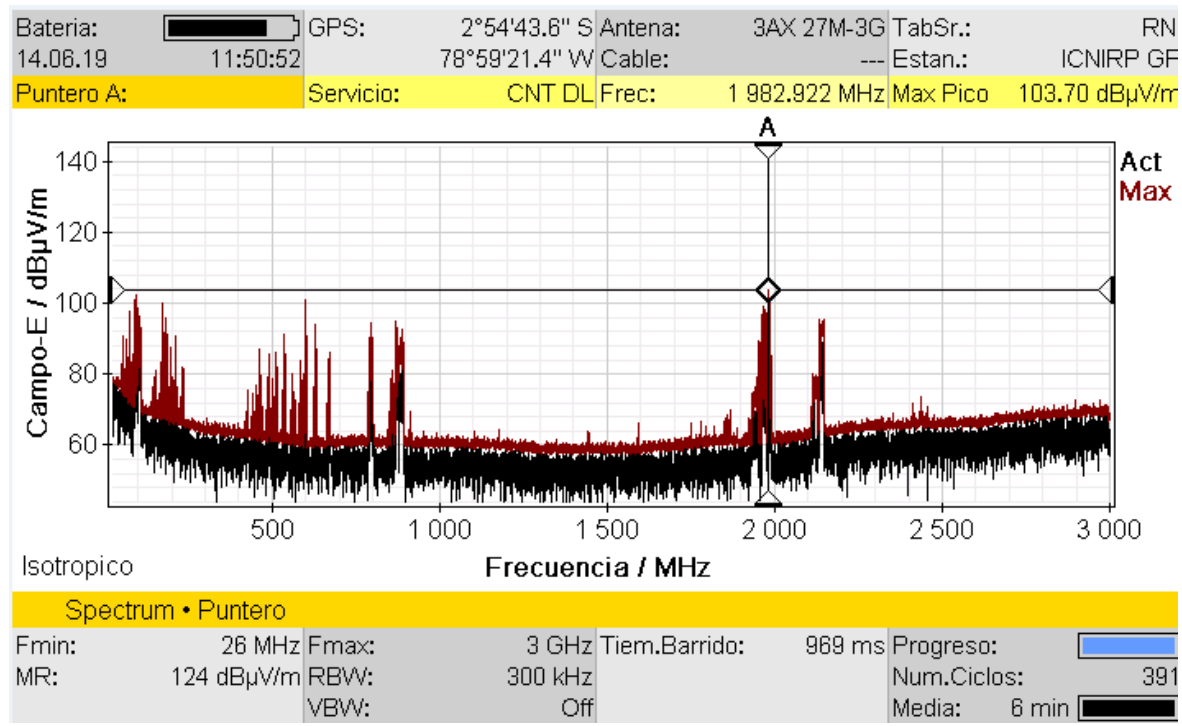

Figura 8. Captura Espectral en EM3

Fuente: Autoría Propia

\begin{tabular}{|c|c|c|c|c|c|}
\hline $\begin{array}{l}\text { Bateria: } \\
14.06 .19\end{array}$ & GPS: & \multicolumn{2}{|c|}{$\begin{array}{l}2^{\circ} 54^{\prime} 43.6^{\prime \prime} \text { S Antena: } \\
78^{\circ} 59^{\prime} 21.4^{\prime \prime} \text { W Cable: }\end{array}$} & $\begin{array}{r}\text { 3AX 27M-3G TabSr.: } \\
\text {--- Estan.: }\end{array}$ & $\begin{array}{r}\text { RNI } \\
\text { ICNIRP GP }\end{array}$ \\
\hline \multicolumn{6}{|c|}{ Tabla de Picos } \\
\hline ndice & Frecuencia & Servicio & Maximo & Activar & \\
\hline 1 & $1982.922337 \mathrm{MHz}$ & CNT DL & $103.70 \mathrm{~dB} \mu \mathrm{V} / \mathrm{m}$ & $-19.89 \mathrm{~dB}$ & \\
\hline 2 & $96.091816 \mathrm{MHz}$ & & $103.00 \mathrm{~dB} \mu \mathrm{V} / \mathrm{m}$ & $-4.88 \mathrm{~dB}$ & \\
\hline 3 & $1981.339235 \mathrm{MHz}$ & CNT DL & $102.19 \mathrm{~dB} \mu \mathrm{V} / \mathrm{m}$ & $-17.71 \mathrm{~dB}$ & \\
\hline 4 & $1981.007770 \mathrm{MHz}$ & CNT DL & $101.42 \mathrm{~dB} \mu \mathrm{V} / \mathrm{m}$ & $-19.82 \mathrm{~dB}$ & \\
\hline 5 & $89.710591 \mathrm{MHz}$ & & $101.07 \mathrm{~dB} \mu \mathrm{V} / \mathrm{m}$ & $-4.50 \mathrm{~dB}$ & \\
\hline 6 & $597.253096 \mathrm{MHz}$ & & $100.87 \mathrm{~dB} \mu \mathrm{V} / \mathrm{m}$ & $-5.79 \mathrm{~dB}$ & \\
\hline 7 & $1982.322641 \mathrm{MHz}$ & CNT DL & $100.78 \mathrm{~dB} \mu \mathrm{V} / \mathrm{m}$ & $-22.63 \mathrm{~dB}$ & \\
\hline 8 & $1983.882175 \mathrm{MHz}$ & CNT DL & $100.54 \mathrm{~dB} \mu \mathrm{V} / \mathrm{m}$ & $-24.21 \mathrm{~dB}$ & \\
\hline 9 & $1981.828685 \mathrm{MHz}$ & CNT DL & $100.35 \mathrm{~dB} \mu \mathrm{V} / \mathrm{m}$ & $-19.81 \mathrm{~dB}$ & \\
\hline 10 & $175.251712 \mathrm{MHz}$ & & $100.18 \mathrm{~dB} \mu \mathrm{V} / \mathrm{m}$ & $-6.02 \mathrm{~dB}$ & \\
\hline 11 & $1965.840724 \mathrm{MHz}$ & CLARO DL & $98.90 \mathrm{~dB} \mu \mathrm{V} / \mathrm{m}$ & $-14.08 \mathrm{~dB}$ & \\
\hline \multicolumn{6}{|c|}{ Isotropico } \\
\hline \multicolumn{6}{|c|}{ Spectrum • Tabla de Picos } \\
\hline \multirow{3}{*}{$\begin{array}{l}\text { Fmin: } \\
\text { MR: }\end{array}$} & \multirow{3}{*}{$\begin{array}{r}26 \mathrm{MHz} \text { Fmax } \\
124 \mathrm{~dB} \mu \mathrm{V} / \mathrm{m} \text { RBW } \\
\text { VBW: }\end{array}$} & \multirow{3}{*}{\multicolumn{2}{|c|}{$\begin{array}{r}3 \mathrm{GHz} \mathrm{T} \\
300 \mathrm{kHz} \\
\mathrm{Off}\end{array}$}} & \multirow{3}{*}{$\begin{array}{l}969 \text { ms Progreso: } \\
\text { Num.Ciclos: } \\
\text { Media: } \quad 6 \text { min }\end{array}$} & \\
\hline & & & & & 418 \\
\hline & & & & & \\
\hline
\end{tabular}

Figura 9. Evaluación del Espectro en el rango de $27 \mathrm{MHz}$ a $3 \mathrm{GHz}$ en EM3

Fuente: Autoría Propia 
Para este sitio se recomienda utilizar el servicio de la operadora CNT E.P. por ser la que posee mayores niveles de intensidad de campo lo que garantiza una buena cobertura

\section{Implementación.}

\section{Hardware.}

A continuación se muestra él diseño de la estación de monitoreo de variables medioambientales y el hardware a utilizar, que permitirán proporcionar los valores que evaluaran la calidad del agua del Rio Yanuncay.

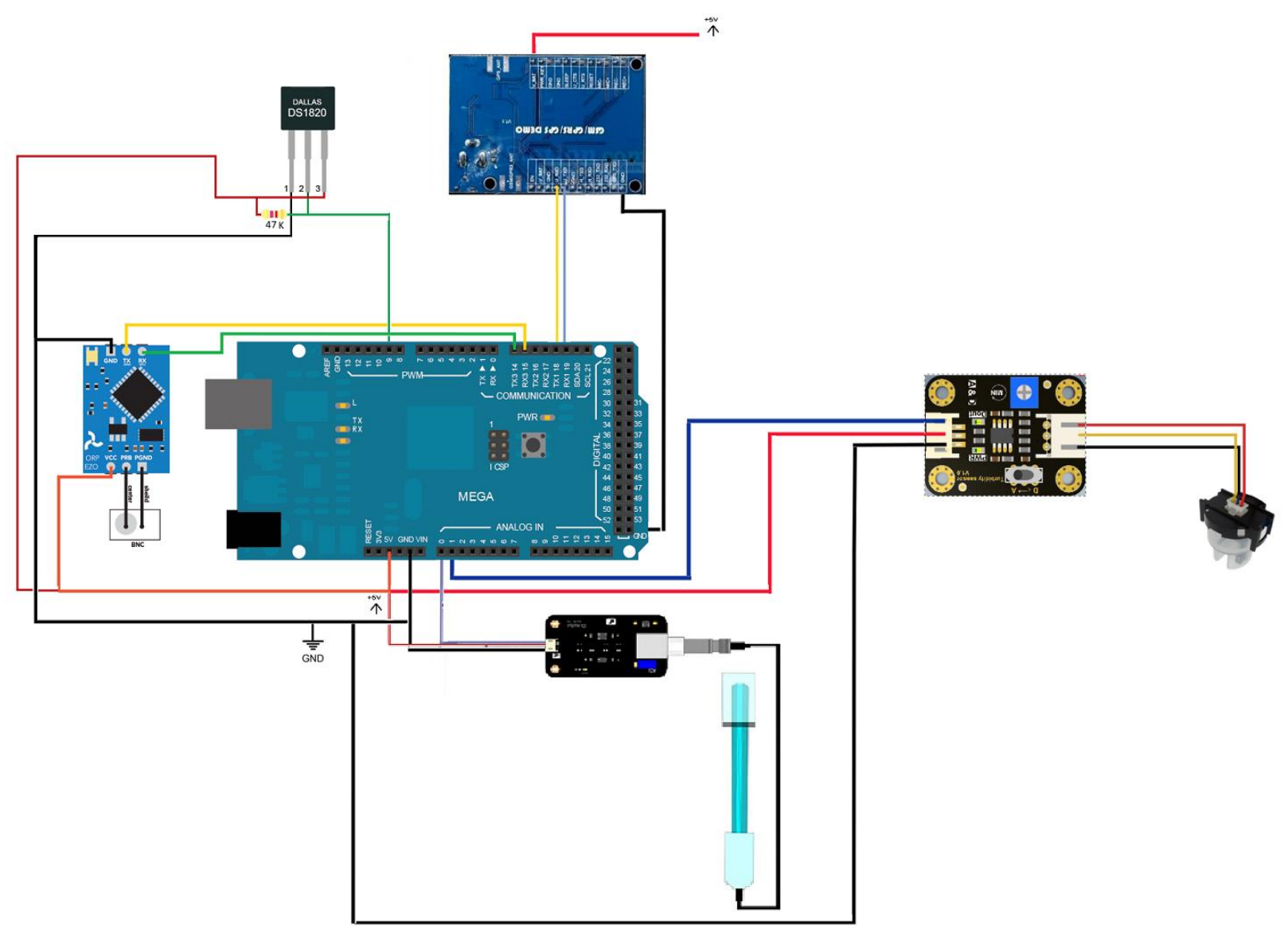

Figura 10. Proceso de adquisición y almacenamiento de la información obtenida Fuente: Autoría Propia 


\section{Software}

El software utilizado para la generación del código a implementar es el entorno de desarrollo integrado (IDE) de Arduino, que es una aplicación que puede ser ejecutada en varias plataformas o sistemas operativos, creada en lenguaje de programación java, admite lenguajes de programación que se basa en el lenguaje estándar $\mathrm{C}++$, para el presente trabajo se tomó como referencia los códigos dados por cada uno de los fabricantes de cada uno de los sensores.

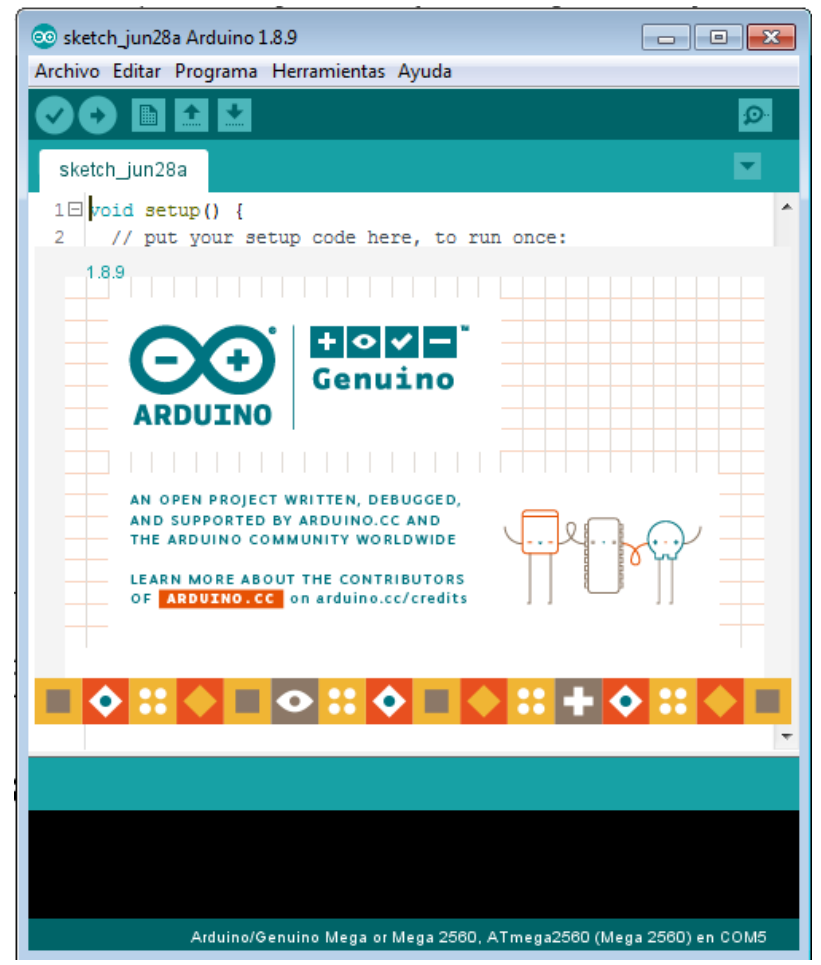

Figura 11. Interfaz del IDE de Arduino.

Para la gestión y almacenamiento en la nube se utiliza la plataforma IO (Thinger.io), en la versión free, esta permite la creación de cuadros de mando para la visualización del comportamiento de cada una de las variables, en general se puede gestionar parámetros que corresponden a transmisión de los datos. 
Armando D. Chulde-Fuentes; Javier B. Cabrera-Mejía
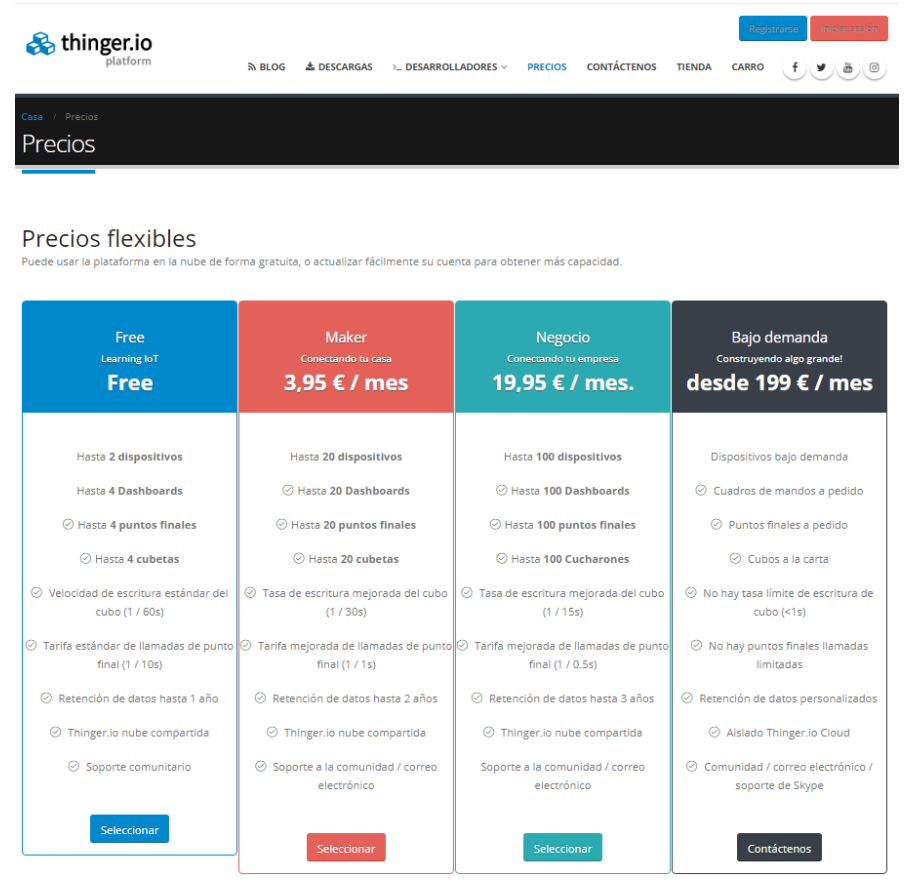

Figura 12. Interfaz del IDE de Arduino.

Fuente: (thinger.io, 2019)

De acuerdo a la metodología planteada, una vez que se han realizados las etapas de análisis del espectro y de los diferentes componentes de hardware a ser utilizadas para la construcción de la estación de monitoreo y su código, se construye dando como resultado un medidor multiparámetro que operaria como una estación de monitoreo de parámetros medioambientales, proporcionando datos en tiempo real.
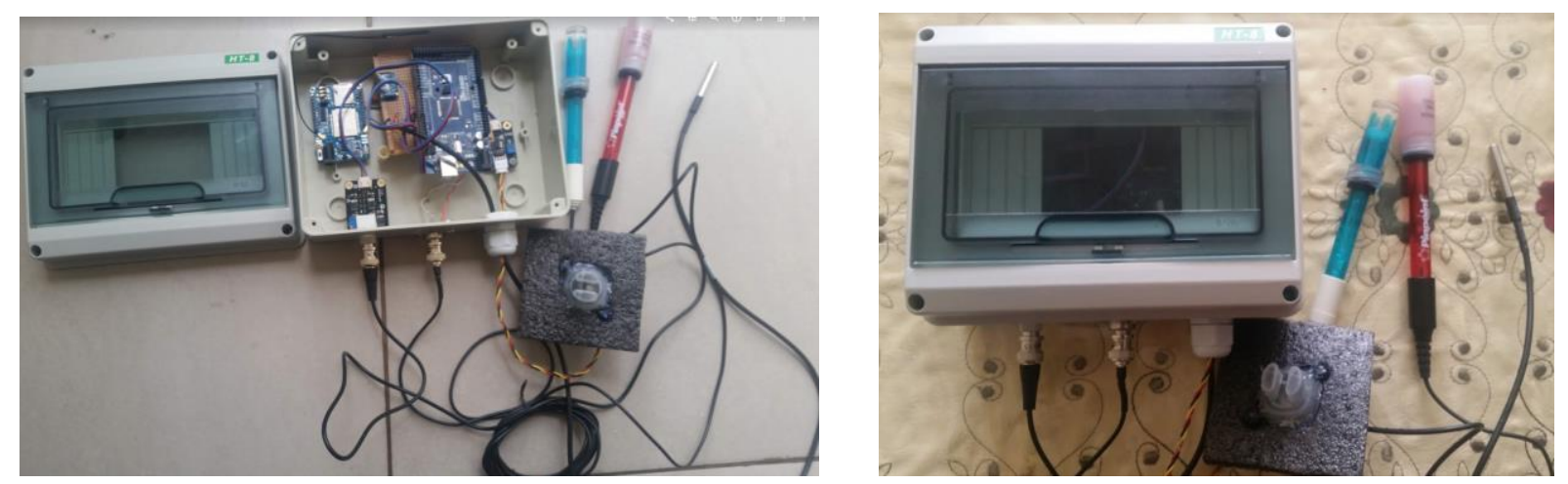

Figura 13. Prototipo de la estación de monitoreo implementada

Fuente: Autoría Propia 
Se realizan pruebas para poder observar el comportamiento de los sensores y poder determinar si existen valores atípicos, por lo que se realizan pruebas tomando como muestra agua de la llave y se le deja por un periodo de $12 \mathrm{~h}$, además se realiza una calibración, especialmente del $\mathrm{pH}$ y el medidor de turbidez tomando como patrones de referencias valores dados por equipos y dispositivos que miden cada parámetro a estudiar y por lo indicado por cada fabricante

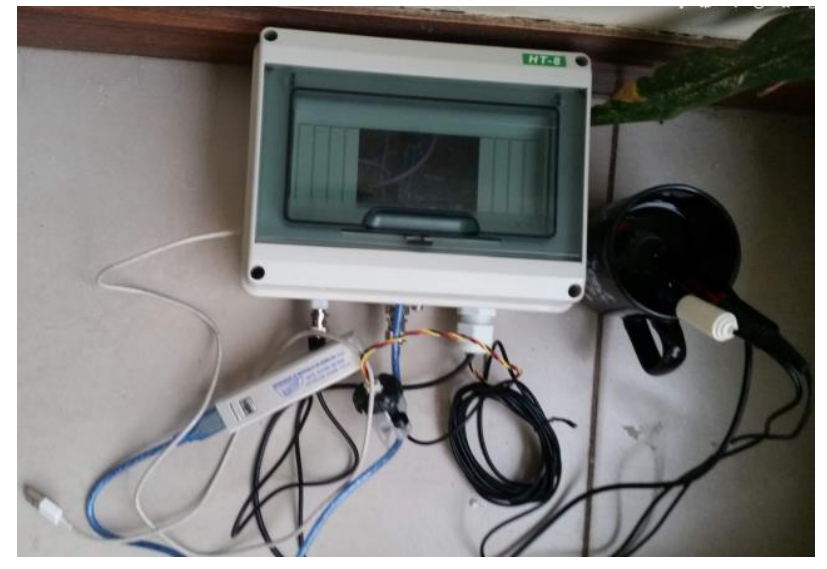

Figura 14. Mediciones efectuadas en ambiente controlado Fuente: Autoría Propia

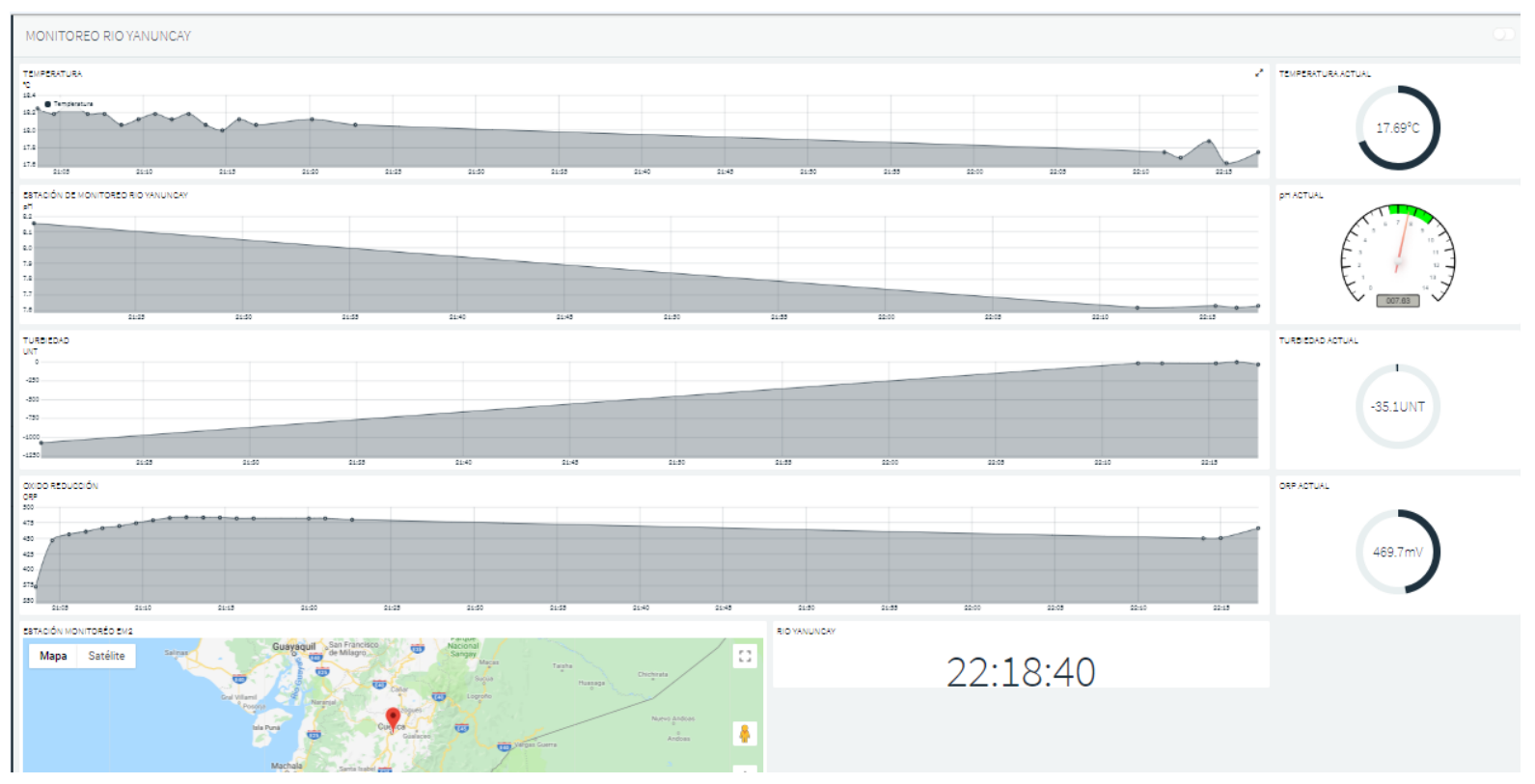

Figura 15. Datos obtenidos en la plataforma IO

Fuente: Autoría Propia 
Una vez que se verifica que los sensores se encuentran transmitiendo datos de una manera adecuada, se realizan pruebas en campo, tomando datos en el punto uno detallado en la tabla 3 ,

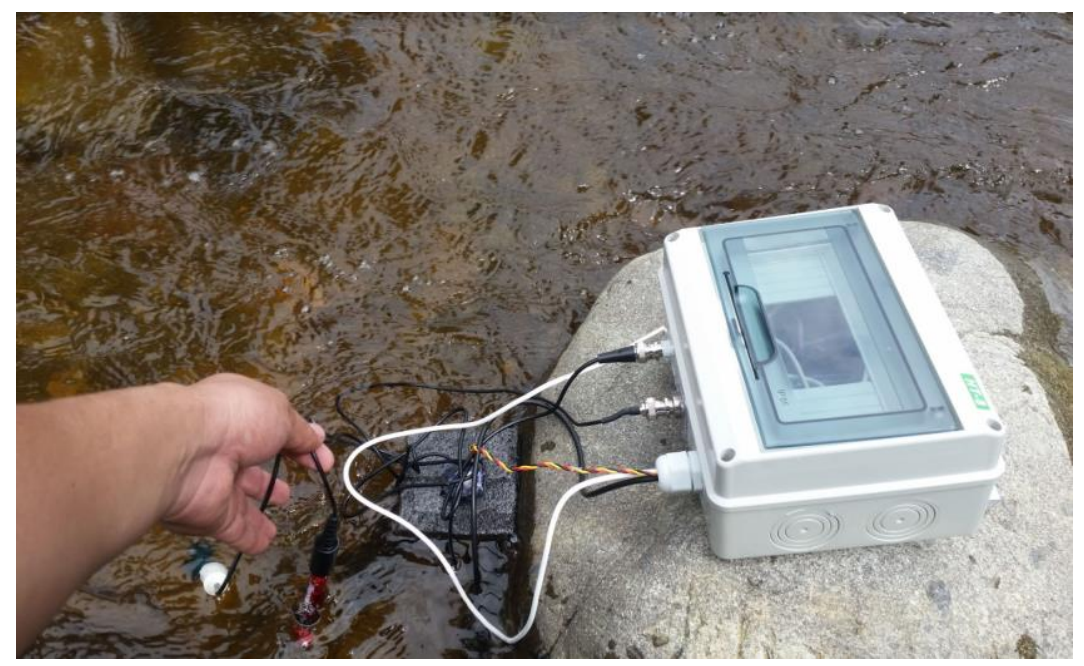

Figura 16. Mediciones efectuadas en campo, punto EM1

Fuente: Autoría Propia

De las mediciones realizadas en el campo se pudo obtener los siguientes resultados:

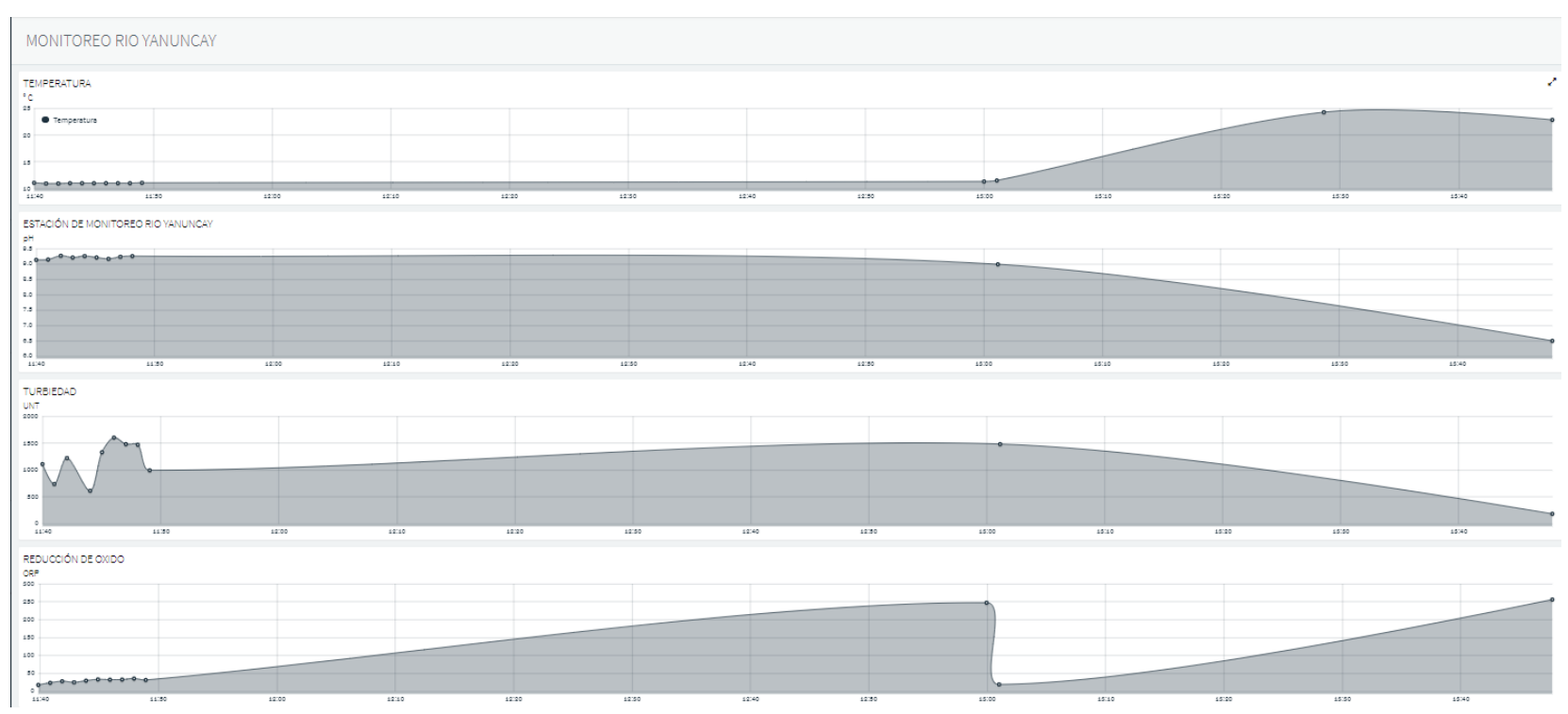



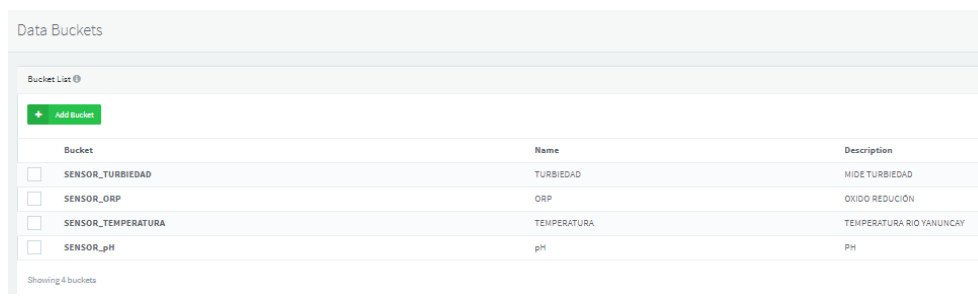

Figura 17. Resultados de medición en punto EM1.- Plataforma IO

Fuente: Autoría Propia

Tabla 4. Datos obtenidos en 10 minutos de monitoreo

\begin{tabular}{|c|c|c|c|c|}
\hline FECHA MUESTRA & $\begin{array}{c}\text { TURBIEDAD } \\
(\mathrm{NTU})\end{array}$ & $\begin{array}{c}\text { OXIDO-REDUCCION } \\
(\mathrm{mV})\end{array}$ & $\begin{array}{c}\text { TEMPERATURA } \\
\left({ }^{\circ} \mathrm{C}\right)\end{array}$ & pH \\
\hline 2019-06-30T11:40:52.071 & -35 & 18.0 & 11 & 9,1 \\
\hline 2019-06-30T11:41:52.254 & -191 & 23.9 & 11 & 9,1 \\
\hline 2019-06-30T11:42:52.735 & -191 & 28.3 & 11 & 9,3 \\
\hline 2019-06-30T11:43:52.912 & -35 & 25.4 & 11 & 9,2 \\
\hline 2019-06-30T11:44:53.390 & -191 & 29.9 & 11 & 9,3 \\
\hline 2019-06-30T11:45:53.793 & -35 & 33.4 & 11 & 9,2 \\
\hline 2019-06-30T11:46:54.291 & -191 & 32.7 & 11 & 9,2 \\
\hline 2019-06-30T11:47:54.831 & -191 & 33.0 & 11 & 9,2 \\
\hline 2019-06-30T11:48:55.250 & -191 & 35.8 & 11 & 9,3 \\
\hline 2019-06-30T11:49:55.912 & -191 & 31.8 & 12 & 9,0 \\
\hline
\end{tabular}

Fuente: Autoría Propia

De los datos obtenidos se puede indicar que al momento del monitoreo realizado el agua se encuentra cristalina, a una temperatura de 11 grados centígrados, un $\mathrm{pH}$ dentro de lo establecido en la norma considerando que este parámetro es inversamente proporcional a la temperatura, se toma la muestra a temperatura ambiental 17 grados centígrados y se tiene una medición de 6.75 de $\mathrm{pH}$, por lo que este parámetro está dentro de lo establecido en la tabla 1; el valor de Oxido reducción nos indica que el agua no está desinfectada por lo que en ella pueden estar presente bacterias e incluso virus.

Los datos recolectados por los diferentes sensores de la estación de monitoreo permitirán a los científicos, entidades gubernamentales, estudiantes y personas que tengan interés en la preservación del agua de los ríos, tener una base de datos de los niveles de contaminación del agua (histórico), datos 
en tiempo real que dará información sobre los niveles de contaminación en puntos específicos del cauce del Río Yanuncay,

Se podrá generar nuevas políticas en favor del manejo y preservación del agua de los ríos de la ciudad de Cuenca y sobre todo un control permanente.

\section{Conclusiones.}

Con la implementación de estaciones de monitoreo de medidores de variables medioambientales diseñadas con elementos de bajo costo, ubicados en puntos estratégicos a lo largo del cauce del Río Yanuncay de la ciudad de Cuenca, permitirá obtener datos confiables y en tiempo real, para que los entes de control y población en general esté informada y conozca la situación real de los niveles de contaminación existentes. Además, que con este tipo de proyectos de investigación se puede impulsar a que la ciudad de Cuenca sea un modelo de ciudad inteligente para el resto de ciudades del Ecuador.

Las acciones que se tomen para la preservación y control de la calidad del agua garantizan una mejor calidad de vida para la población de la ciudad de Cuenca, y un ahorro considerable en el tratamiento del agua para consumo humano.

El presente trabajo de investigación fue desarrollado con miras a ser escalable y que se lo pueda implementar y replicar con ayuda los departamentos de investigación de la Universidad Católica de Cuenca, para que se evalué la calidad del agua de los ríos que atraviesan la ciudad, y poder determinar los niveles de contaminación, para lo cual se medirán los parámetros en un punto inicial del cauce (antes de que atraviese la ciudad) y otro punto será al atravesar la ciudad, con el objetivo de que se tenga un control adecuado por parte de los entes de regulación tanto a nivel estatal (Ministerio del Ambiente) como regional (GAD de Cuenca).

\section{Bibliografía.}

Agencia de Protección Ambiental de Estados Unidos. (noviembre de 2018). Lower Merrimack River en Español. Recuperado el 17 de abril de 2019, de Datos en Directo Sobre la Calidad del Agua de la Cuenca Baja del Río Merrimack: https://espanol.epa.gov/eslowermerrimackriver/datosen-directo-sobre-la-calidad-del-agua-de-la-cuenca-baja-del-rio-merrimack\#Temperatura 
Implementación de un prototipo de estación de monitoreo (EM) para el análisis de variables medioambientales. Caso de estudio: Río Yanuncay de la ciudad de Cuenca

Amazon.com. (2019). ELEGOO MEGA 2560 R3 Board ATmega2560 ATMEGA16U2 + Cable USB Compatible con Arduino Azul. Obtenido de https://www.amazon.com/gp/product/B01H4ZLZLQ/ref=ppx_od_dt_b_asin_title_s00?ie=U $\mathrm{TF} 8 \& \mathrm{psc}=1$

Arce Moncada, M. F., \& Leiva Calderón, M. A. (2009). DETERMINACIÓN DE LA CALIDAD DE AGUA DE LOS RÍOS DE LA CIUDAD DE LOJA Y DISEÑO DE LÍNEAS GENERALES DE ACCIÓN PARA SU RECUPERACIÓN Y MANEJO. Loja, Ecuador: Universidad Técnica Particular de Loja.

El-Khouri Vidarte, N. (2016). Adaptación e implementación de un sistema autónomo de bajo coste de monitorización de calidad del agua en tiempo real.

Organización Mundial de la Salud. (10 de febrero de 2017). Agua, saneamiento e higiene. Obtenido de https://www.who.int/water_sanitation_health/es/

Organización Mundial de la Salud. (2018). Organización Mundial de la Salud. Obtenido de https://www.who.int/water_sanitation_health/publications/drinking-water-quality-guidelines4-including-1st-addendum/es/

Organización Mundial de la Salud. (2019). Higiene del saneamiento del agua. Obtenido de https://www.who.int/water_sanitation_health/diseases-risks/diseases/en/

REGISTRO OFICIAL. (s.f.). REFORMA DEL LIBRO VI DEL TEXTO UNIFICADO DE LEGISLACIÓN SECUNDARIA. Norma de Calidad Ambiental y de Descarga de Efluentes: Recurso Agua.

thinger.io. (28 de 06 de 2019). Precios. Obtenido de https://thinger.io/pricing/ 